\title{
SAR IMAGE SIMULATION OF SHIP TARGETS BASED ON MULTI-PATH SCATTERING
}

\author{
Yuhua Guo ${ }^{1 *}$, Haitao Wang ${ }^{1}$, Haiquan $\mathrm{Ma}^{1}, \mathrm{Ke} \mathrm{Li}^{1}$, Zhenghuan Xia ${ }^{1}$, Yan $\mathrm{Hao}^{1}$,Hui Guo ${ }^{1}$, Huifeng Shi ${ }^{1}$, Xiaohan Liao ${ }^{2}$ and \\ Huanyin Yue ${ }^{2}$ \\ ${ }^{1}$ State Key Laboratory of Space-Ground Integrated Information Technology, Beijing Institute of Satellite Information \\ Engineering,No. 55 Zique Road, Haidian district,Beijing 100095, China, yuhuaguo321@163.com \\ ${ }^{2}$ State Key Laboratory of Resources and Environment Information System, Institute of Geographic Sciences and Natural Resources \\ Research, Chinese Academy of Science, No.11,Datun Road, Chaoyang District, Beijing 100101,China
}

\section{Commission III, WG III/2}

KEY WORDS: Ships, Synthetic Aperture Radar(SAR), Imaging simulation, Multipath scattering, Feature Ellipse

\begin{abstract}
:
Synthetic Aperture Radar (SAR) plays an important role in the classification and recognition of ship targets because of its all-weather working ability and fine resolution. In SAR images, besides the sea clutter, the influence of the sea surface on the radar echo is also known as the so-called multipath effect. These multipath effects will generate some extra "pseudo images", which may cause the distortion of the target image and affect the estimation of the characteristic parameters. In this paper,the multipath effect of rough sea surface and its influence on the estimation of ship characteristic parameters are studied. The imaging of the first and the secondary reflection of sea surface is presented. The artifacts not only overlap with the image of the target itself, but may also appear in the sea near the target area. It is difficult to distinguish them, and this artifact has an effect on the length and width of the ship.
\end{abstract}

\section{MANUSCRIPT}

\subsection{General Instructions}

SAR imaging mainly uses pulse compression of wideband signal to achieve high resolution of range direction. At the same time, using the relative motion of radar and target, virtual aperture is formed, which is far larger than real aperture. Thus, the high resolution of azimuth direction can be realized by analyzing the Doppler frequency shift of signals from different azimuths. SAR can provide high resolution target images under the condition of all-weather and all-day, which plays an important role in the investigation and surveillance of sea surface ships(Margarit G., 1998; Morse A.J.,1997; Pastina D., 2009).

When the ship above the sea surface is observed by radar, there are many propagation paths between the target scattering center and radar due to the scattering of the sea surface.In addition to direct echoes between the ship and the radar, the electromagnetic waves returned to the radar by other paths will create some additional "artifacts" in the radar imaging results. In order to study the effect of multipath effect on the radar image of a ship, a common method is to treat the sea surface as a horizontal plane with a certain reflection coefficient, and through the geometric analysis of the propagation path. The functional relationship between the multipath propagation delay and the target scattering center height, the radar position and the angle of view is found, and the position coordinates of the multipath artifacts are determined(Liu H. W., 2000; Berizzi F.,1998; Garren D. A.,2008) . But the assumption of horizontal sea surface is not always true. In order to study the multipath propagation effect in rough surface environment, electromagnetic scattering modeling is usually needed, especially the object-rough surface composite modeling technique. A generalized forward and backward method is used to simulate the radar cross section of a 2-D ship model over the sea surface of PM spectrum under different wind speeds (Burkholder R. J., 2001). A simulation method for the signal characteristics of ship target moving radar on time - varying sea surface has been studied (Xu X.J., 2011). He S. Y. (2009) has applied electromagnetic scattering modeling technology to the morphological analysis of high resolution range profiles of targets in multipath environments.

Under the condition of medium rough sea surface, there is not only overlap between artifact and ship target imaging itself, but also the appearance of sea surface adjacent to the ship target area. The multipath artifacts of primary reflection are studied (Duan C.W., 2013), but the imaging of secondary reflection of sea surface is ignored.In this paper, we consider the imaging of the first and the secondary reflection of sea surface.

\section{THE MULTIPATH ARTIFACT}

\subsection{Four-path model}

In this part, we mainly review the main conclusions given in the reference (Duan C.W., 2013). The electromagnetic propagation between the scattering center and the radar above the horizontal sea surface can be represented by the four-path model shown in figure 1.The target scattering center, radar and sea surface reflection point are represented by S,R and F. The electromagnetic wave that reaches the target can be either directly reached by $\mathrm{R}$ or reflected through the $\mathrm{F}$ point, along the $R F \rightarrow F S$ path of propagation. Similarly, the target scattering electromagnetic wave can return to radar via SR and $S F \rightarrow F R$ paths. That is to say, there are four propagation paths between radar and target:1. $R S \rightarrow S R$, 2. $R S \rightarrow S F \rightarrow F R, 3 . R F \rightarrow F S \rightarrow S R$, 
4. $R F \rightarrow F S \rightarrow S F \rightarrow F R$,in which the path 1 is called direct echo, the paths 2 and 3 are called the first reflection echo, and the second reflection echo is passed through 4.

Suppose the radar emission signal is $h(\tau)$ and $|R S|=R$, then the echo signal generated by the direct arrival wave is:

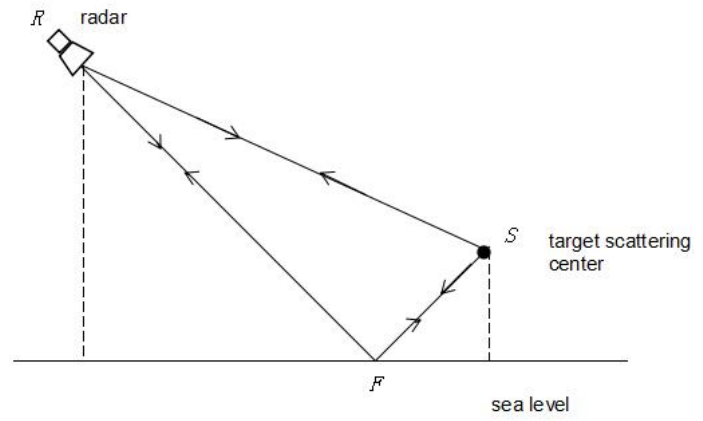

Figure 1. A four-path model for multipath propagation of electromagnetic waves on horizontal sea surface

$$
S(\tau)=A \cdot S\left(\tau-\frac{2 R}{c}\right)
$$

where $\mathrm{A}$ is the scattering coefficient of the scattering center. After considering the multipath propagation, the echo signal can be written as:

$S S(\tau)=S(\tau)+2 \rho S\left(\tau-\frac{\Delta r}{c}\right)+\rho^{2} S\left(\tau-\frac{2 \Delta r}{c}\right)$

The two terms on the right of the equal correspond to the first and second reflection echoes due to the wave process phase of path 2 and 3, respectively. $\Delta r=|R F|+|F S|-|R S|$

represents the delay of a single reflection multipath echo relative to a direct echo. $\rho$ is the reflection coefficient of horizontal sea surface.

In the echo signal, in addition to the peak value formed by the direct wave of each scattering center, the latter two terms of equation (2) may also form strong scattering in the image, thus producing the so-called multipath artifacts.These artifacts are similar to the real target shape, but the position shifts and the amplitude changes accordingly. The coordinate offset is determined by $\Delta r$, and the variation of the amplitude is related to the sea surface reflection coefficient.

In order to investigate the effect of the multipath effect on the target SAR image in the horizontal sea surface, the target coordinate system is set up as shown in Figure 2. Suppose the radar moves in the direction indicated by the arrow, and at the same time illuminates the ship target in the positive sidelooking mode. The radar beam is directed to the target origin o as the slow time zero, and the distance between the radar and o is $R_{B}$. The azimuth and pitch angle of radar are $\theta_{m}$ and $\varphi_{m}$ respectively at the time of slow time $t_{m}$. In the target coordinate system, the radial distance of the $\mathrm{k}$-th scattering center on the ship relative to the radar is obtained in the target coordinate system as follows (Duan C. W., 2013):

$$
\begin{aligned}
R_{k}\left(t_{m}\right)= & R_{0}\left(t_{m}\right)-x_{k} \cos \theta_{m} \cos \varphi_{m} \\
& -y_{k} \sin \theta_{m} \cos \varphi_{m}-z_{k} \sin \varphi_{m}
\end{aligned}
$$

where $R_{0}\left(t_{m}\right)$ is the radial distance of o relative to radar:

$$
R_{0}\left(t_{m}\right)=\sqrt{R_{B}^{2}+\left(v t_{m}\right)^{2}} \approx R_{B}+\frac{\left(v t_{m}\right)^{2}}{2 R_{B}}
$$

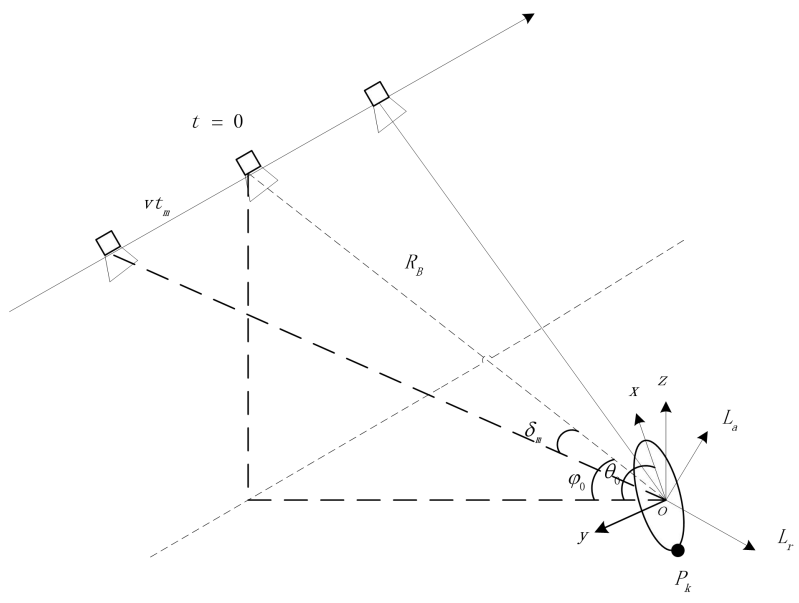

Figure 2. SAR imaging scene model

In the case of a small change in the overall angle of view $\delta$, we can obtain $R_{k}\left(t_{m}\right) \approx A_{k}+B_{k} t_{m}+C_{k} t_{m}^{2}$, where

$A_{k}=R_{B}-\left(x_{k} \cos \theta_{0}+y_{k} \sin \theta_{0}\right) \cos \varphi_{0}-z_{k} \sin \varphi_{0}$

$$
B_{k}=\left(x_{k} \sin \theta_{0}-y_{k} \cos \theta_{0}\right) \frac{v}{R_{B}}
$$

$$
C_{k}=\left[R_{B}+\frac{\left(x_{k} \cos \theta_{0}+y_{k} \sin \theta_{0}\right)}{\cos \varphi_{0}}\right] \frac{v^{2}}{2 R_{B}^{2}}
$$

By SAR imaging, the azimuth and range coordinates of scattering points in SAR images are as follows:

$$
\begin{gathered}
X_{k}=-\frac{R_{B}}{V} B_{k} \\
Y_{k}=A_{k}-B_{k}
\end{gathered}
$$


It can be seen from Figure 1 that the wave path of direct echo 1 is $2 R_{k}\left(t_{m}\right)$, and that corresponding to paths 2 and 2 is $2 R_{k}\left(t_{m}\right)+\Delta r_{k}\left(t_{m}\right)$. Here, the amount of multipath delay $\Delta r_{k}\left(t_{m}\right)$ is related to slow time $t_{m}$.

The multipath delay can be written as follows through the Taylor expansion :

$$
\Delta r_{k}\left(t_{m}\right) \approx a_{k}+b_{k} t_{m}+c_{k} t_{m}^{2}+o\left(t_{m}^{2}\right)
$$

The coefficients are determined by the parameters of the scene. If the focus is good, the multipath echo reflected by the sea surface will be imaged at the position of the imaging distance and azimuth direction of the corresponding direct wave, $D_{r}=a_{k} / 2$ and $D_{a}=R_{B} b_{k} / 2 v$, respectively. The imaging position of the secondary emitter echo is $2 D_{r}$ and $2 D_{a}$ apart from the direct wave.

\section{THE INFLUENCE OF ARTIFACES ON THE ESTIMATION OF SHIP CHARACTERISRIC PARAMETERS}

\subsection{Imaging of a single reflection of the sea surface}

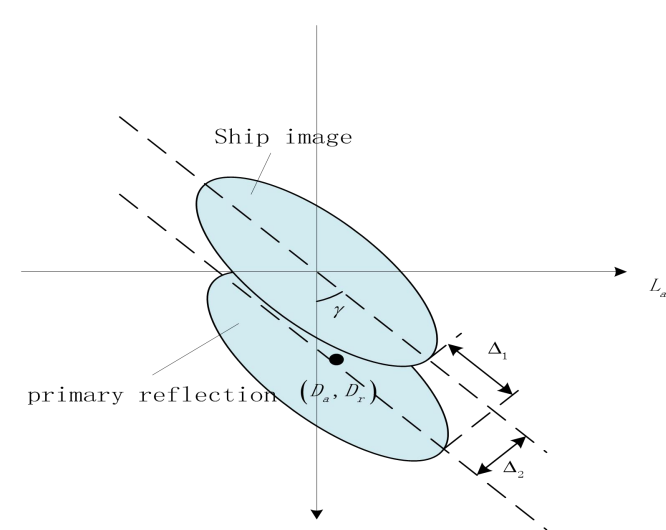

Figure 3.Simplified model of ship image and its primary artifacts

As shown in Figure 3, on the radar imaging plane, an ellipse with a center at the origin, a long and a short half axis A and B, and an obliquity approximates the ship image region, and assumes that the multipath pseudo-image reflected once by the sea surface is also such an ellipse. But the center is shifted to $\left(D_{a}, D_{r}\right)$.So the ship image obtained by SAR is actually superposed by the above two ellipses. We assume that the change of attitude angle of the superposition object can be ignored. The effect of multipath artifact on ship image is that the length of ship increases $\Delta_{1}$ and the width increases $\Delta_{2}$.

$$
\left(\begin{array}{c}
\Delta_{1} \\
\Delta_{2}
\end{array}\right)=\left[\begin{array}{cc}
-\sin \gamma & \cos \gamma \\
\cos \gamma & \sin \gamma
\end{array}\right]\left[\begin{array}{c}
D_{a} \\
D_{r}
\end{array}\right]
$$

\subsection{Imaging of sea surface secondary reflection}

If the focus is good, the center position of the secondary reflection echo of the sea surface is shifted to $\left(2 D_{a}, 2 D_{r}\right)$, then

$$
\left(\begin{array}{l}
\Delta_{1}^{\prime} \\
\Delta_{2}^{\prime}
\end{array}\right)=\left[\begin{array}{cc}
-\sin \gamma & \cos \gamma \\
\cos \gamma & \sin \gamma
\end{array}\right]\left[\begin{array}{c}
2 D_{a} \\
2 D_{r}
\end{array}\right]=2\left(\begin{array}{c}
\Delta_{1} \\
\Delta_{2}
\end{array}\right)
$$

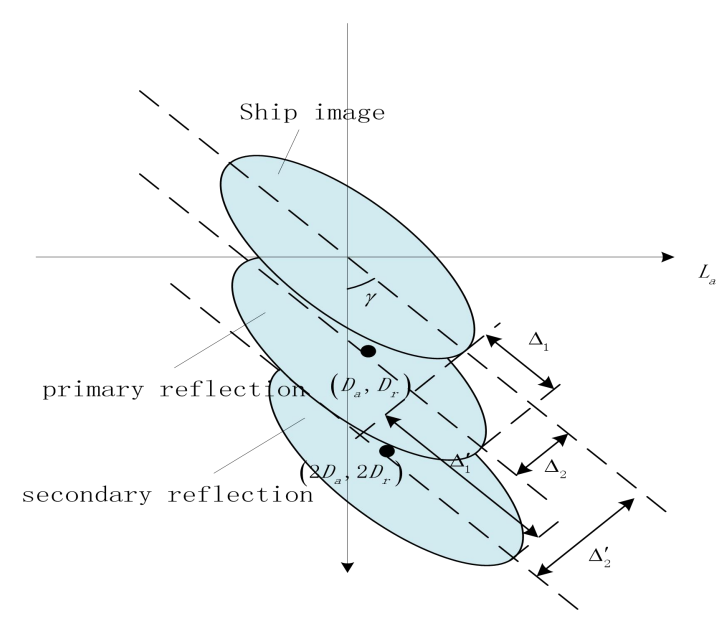

Figure 4.Simplified model of ship image and its secondary reflection artifacts

Therefore, the ship image obtained by SAR is actually made up of three ellipses. As shown in Figure 4, the effect of multipath artifacts on the ship's image is to increase its length by $\Delta_{1}^{\prime}$, and the width of the ship is increased by $\Delta^{\prime}$. The probability density function $\left(\Delta_{1}^{\prime}, \Delta_{2}^{\prime}\right)$ can be deduced from the joint probability density function $\left(D_{a}, D_{r}\right)$. For example, if the scattering point distribution can be approximated to a two-dimensional normal distribution, it is easy to know that $\left(\Delta_{1}^{\prime}, \Delta_{2}^{\prime}\right)$ can be approximated to a two-dimensional normal distribution.

\section{CONCLUSION}

In this paper, the effect of multipath propagation on ship image and its characteristic parameter estimation is studied, and the imaging results of the secondary reflection of the sea surface are given . The mathematical expression of the influence of pseudo images on the length and width of ship is presented.

\section{REFERENCES}

Margarit G., Tabasco A.,1998. Ship Classification in Single-Pol SAR Images of Ships. SPIE Proc. \#3371, Conference Automatic Target Recognition VIII, Orlando.

Morse A. J., Protheroe M. A.,1997. Vessel Classification as Part of An Automated Vessel Traffic Monitoring System Using SAR Data. International Journal of Remote Sensing.18(13), pp. 2709-2712.

Pastina D., Spina C., 2009. Multi-feature Based Automatic Recognition of Ship Targets in ISAR.IET Radar, Sonar and Navigation, 3(4), pp.406-423. 
Liu H. W., Su H. T.,Shui P. L.,et al. 2000.Multipath signal resolving and time delay estimation for high range resolution radar. Proc. of IEEE International Radar Conference. Virginia: IEEE Press, pp. 497-502.

Berizzi F., Diani M.,1998. Multipath effects on ISAR image reconstruction. IEEE Trans.Aerospace and Electronic System, 34(2), pp. 645-653.

Garren D. A., Goldstein J. S., Obuchon D. R., et al. 2008.SAR image formation algorithm with multipath reflectivity estimation.Proc, of IEEE International Radar Conference, Philadelphia: IEEE Press, pp.323-328.

Burkholder R. J.,Pino M. R., Obelleiro F.,2001. A M onte Carlo Study of the Rough-sea-surface Influence on the Radar Scattering from Two-dimensional Ships. IEEE Antennas and Propagation Magazine,43(2), pp. 25-33.

Xu X.J., Jiang D., Li X.F., 2011. Modeling of Dynamic Radar Signatures for Ships on Time-varying Sea Surface, Systems Engineering and Electronics, 33(1), pp. 42-47.

He S. Y.,Deng F. S., Chen H. T., et al,2009. Range Profile Analysis of the 2-D Target Above a Rough Surface Based on the Electromagnetic Numerical Simulation. IEEE Trans. Antennas and Propagation,57(10), pp. 3258-3263.

Duan C.W., 2013. Feature Parameter Estimation of Sea Ships Based on SAR Imaging. National University of Defense Technology.

Revised March 2018 